\title{
A PRIMER TO METHOD ENGINEERING
}

\author{
J. J. Odell \\ James Odell Associates \\ 1315 Hutchins Avenue \\ Ann Arbor, MI 48103 USA \\ Tel: +1 313 994-0844 \\ email:71051.1733@compuserve.com
}

\section{INTRODUCTION}

A methodology is a body of methods employed by a discipline.

A method is a procedure for attaining something.

Method engineering is the coordinated and systematic approach to establishing work methods.

Traditional methodologies for information system (I.S.) development are—by nature-general purpose. As such, they contain an ideal set of methods, techniques, and guidelines that in reality can never be followed literally. They must be tuned to the situation at hand. Steps are sometimes omitted, added, or modified. Guidelines are often modified or ignored to fit special circumstances, such as technology, development expertise, the application, and external factors. [Harmsen, 1994]

To complicate things further, numerous methodologies exist for I.S. development-each with its own set of tools and techniques. Comparing and selecting an approach from a multitude of methodologies is confusing and difficult. To aid in this selection, various comparison standards have been proposed for object-oriented methodologies, such as those documented by the OMG [Hutt, 1994a; 1994b]. Some approaches attempt to harmonize several methodologies - forming yet another rigid methodology [Coleman, 1994]. Other methodologies provide a choice of options, or paths, that the user can select depending on the circumstances. In short, an I.S. project can choose from three basic methodologies, as depicted in Fig. 1. 


\begin{tabular}{|l|l|l|}
\hline $\begin{array}{l}\text { Flexibility } \\
\text { : ad hoc development } \\
\text { few guidelines } \\
\text { - no uniform terminology } \\
\text { - project management and measurability } \\
\text { difficult }\end{array}$ \\
$\begin{array}{l}\text { flexible resource allocation } \\
\text { no repository standards }\end{array}$
\end{tabular}

Figure 1 Methodological approaches fall into three categories (adapted from Harmsen [Harmsen, 1994]).

\section{METHOD ENGINEERING}

Flexibility without control can hardly be considered a methodology, since any systematic and coordinated approach to establishing work methods is absent. For such an approach to be systematic and coordinated requires method engineering.

Method engineering produces methodologies. For I.S., a methodology is a body of methods employed to develop automated systems. In turn, a method defines the steps needed to automate a system - along with the required techniques and tools and the anticipated products. Adapting a methodology to the needs of a particular project is sometimes called situational method engineering. For I.S., situational method engineering designs, constructs, and adapts I.S. development methods.

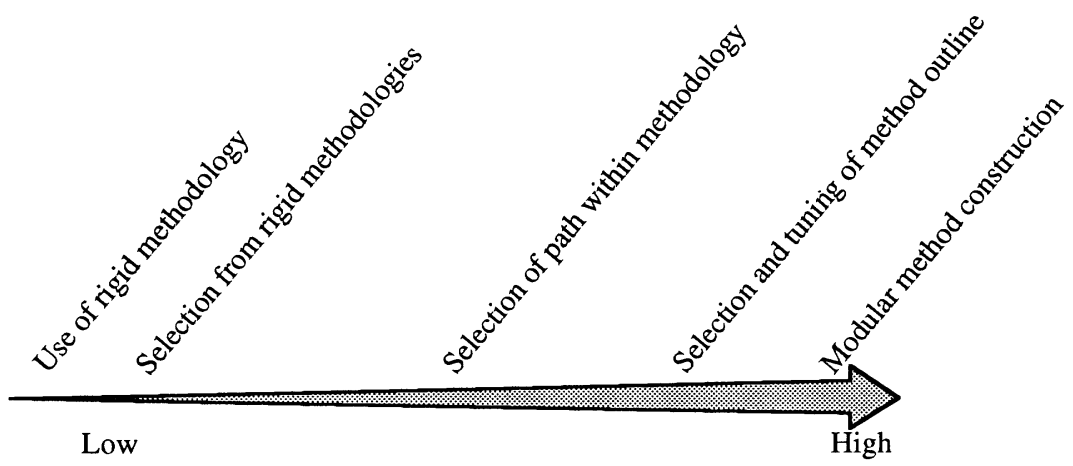

Figure 2 Degrees of flexibility for I.S. situational method engineering (adapted from Harmsen [Harmsen, 1994]).

As indicated in Fig. 2, method engineering has various degrees of flexibility. These are as follows:

- Use of a rigid methodology. At one extreme, using a rigid methodology permits virtually no flexibility. Such methodologies are based on a single development philosophy and thus adopt fixed standards, procedures, and techniques. Project managers are typically not permitted to modify the methodology.

- Selection from rigid methodologies. Instead of permitting only one rigid approach, this option allows each project to choose its methodology from one of several rigid methodologies. This makes possible the selection of an approach that might be more appropriate for the project. However, this is a bit like buying a suit without having it altered. You make the best of what is available, despite the fact that the chosen methodology will probably not fit the project perfectly. Furthermore, each methodology involves additional purchase and training costs. 
- Selection of paths within a methodology. Many methodologies permit more flexibility by providing a choice of predefined paths within the methodology. Typical development paths include traditional and rapid application development. Some methodologies now include paths that support development aspects, such as package selection, pilot projects, client/server, realtime, knowledge-based systems, and object orientation. A common disadvantage, however, is that it may not be possible to combine some options. For instance, realtime, knowledge-based projects may not be supported.

- Selection and tuning of a method outline. This option permits each project to both select methods from different approaches and tune them to the project's needs. Typically, this involves selecting a global method process and data model. These models, then, are further adapted and refined by the project. This option is best supported by an automated tool.

- Modular method construction. One of the most flexible options is to generate a methodology for a given project from predefined building blocks. Each building block is a method fragment that is stored in a method base. Using rules, these building blocks are assembled based on a project's profile. The result is an effective, efficient, complete, and consistent methodology for the project.

An automated tool is recommended for this option. Here, a project's methodology can be generated automatically and then adapted and further refined by the project manager.

Performing the entire activity manually would require much work and time. Such an option is illustrated in Fig. 3.

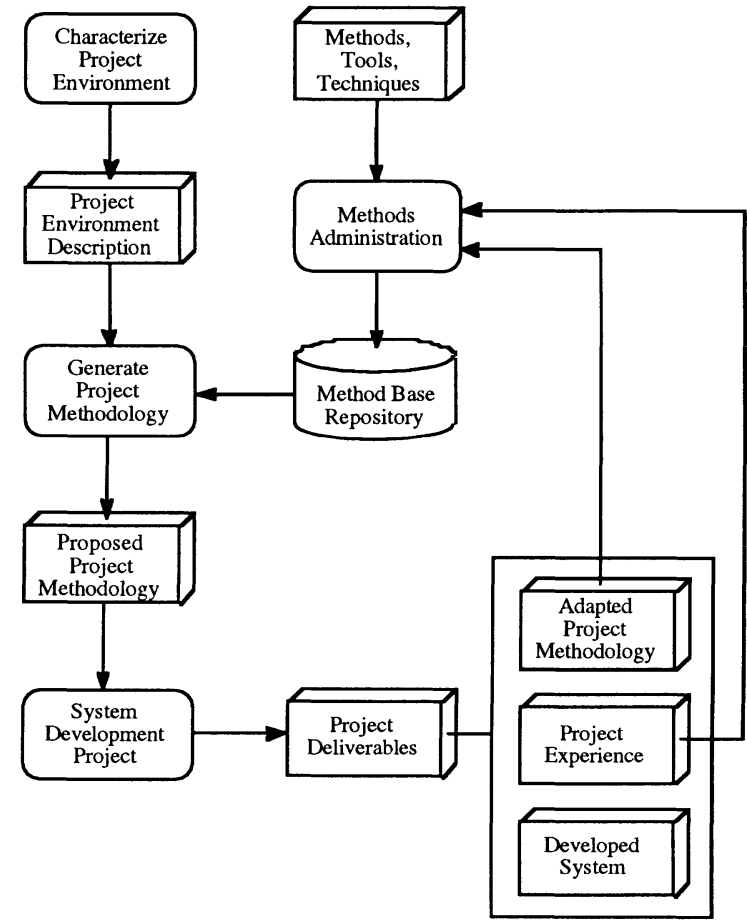

Figure 3 An object-flow diagram specifying the process of modular method construction. 


\section{COMPUTER-AIDED METHOD ENGINEERING}

Computer-Aided Software Engineering (CASE) automates automation. In contrast, ComputerAided Method Engineering (CAME) automates the assembly of methods. A CAME tool should support the following activities [Harmsen, 1994]:

- Definition and evaluation of contingency rules and factors. In order to choose the right method fragments for a project, rules and factors for selecting the proper method fragments must be defined. Method engineers are responsible for these definitions. Given the project profile and method base, the CAME tool selects and assembles the appropriate methodology.

- Storage of method fragments. Selecting and assembling a methodology from method fragments requires a method base. This method base is the repository from which method engineers and the CAME tool can select various method fragments. As new methodologies arise, they can also be incorporated into the method base.

- Retrieval and composition of method fragments. Certainly, for a CAME tool to generate a methodology from a method base, retrieval operations must be available for method fragments. However, total automation of methodology generation may never be completely feasible. A more realistic scenario could involve both automatic generation and a method engineer. The method engineer should be able to manipulate and modify method fragments within a methodology.

- Validation and verification of the generated methodology. The CAME tool should not only support selecting and assembling a methodology, it should also check the results. The tool, therefore, should incorporate guidelines to ensure that the correct set of method fragments has been selected. Furthermore, the tool should ensure that the fragments are assembled in a consistent manner. In other words, the CAME tool should ensure, or assist in ensuring, the quality of the generated methodology. (After all, generated methodologies must meet the same standards as standards methodologies.)

- Adaptation of the generated methodology. The method base should also accumulate the experience of previous projects and their methodologies. This experience should be used to improve method fragments, along with their contingency rules and factors. (Also illustrated in Fig. 4.) In other words, practical experience should be used to adapt future methodologies.

- Integration with a meta-CASE tool. CAME and CASE tools should eventually be integrated. When a methodology is generated for a particular project, the appropriate supporting tools should also be integrated. Adapting a CASE tool in this fashion would require configuring the CASE tool to support the resulting methodology. In other words, a meta-CASE tool would be required so that techniques and diagrammatic representations can be defined based on the methodology. Such a tool would be similar in nature to the CAME tool. Within this meta-CASE tool, CASE fragments would have to be defined. Additionally, it would require the ability to retrieve and compose new conceptual fragments.

- Interface with a method base. This method base is the repository for the various method fragments from which method engineers and the CAME tool can select.

To support CAME, the I.S. organization requires two additional roles-the method engineer and the method administrator. The method administrator is responsible for the contents of the method base. The method engineer is responsible for generating the right methodology for each project. Both support and are supported by the CAME tool-and are part of a larger framework called process management.

CAME tools are being developed by many organizations around the world. They are currently available from companies such as, James Martin \& Co and Ernst \& Young. While still in their infancy, the CAME tools from these two companies support many of the properties described above. 


\section{PROCESS MANAGEMENT}

To support applications systems, the repository must-of course - contain information about the product of I.S. development. This includes information regarding analysis results, such as structural and behavioral models, business rules, and so on. For design and implementation, the repository would include information such as design templates, application data structures, programs, and interfaces. Additionally, the development repository must also contain processrelated information, such as intermediate results, human agents, tools involved, process plans, design decisions, and steps taken to execute them.

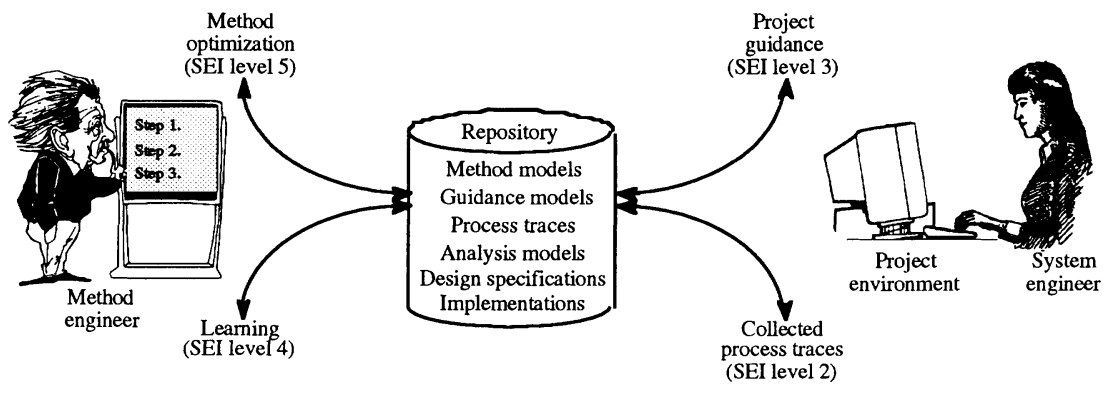

Figure 4 An environment for process management.

\section{SEI support}

The Software Engineering Institute (SEI) has been influential in the movement toward highquality products. Its framework proposes five levels of process maturity: initial, repeatable, defined, managed, and optimizing [Paulk, 1993]. This same framework can be applied to process management.

Jarke recommends several kinds of SEI-related actions be performed that will ensure a high-quality process management environment [Jarke, 1994]. These are illustrated in Fig. 4. At the initial level, an organization does not provide a stable environment. Here, no repository exists. At the repeatable level, policies for managing a project and procedures to implement those policies are established. The planning and management of new projects is based on experience with similar projects. This is aided by capturing process traces, as indicated in the lower right of Fig. 4. At the defined level, an organization standardizes both its system engineering and management processes. Such an organization exploits effective softwareengineering practices when standardizing its processes. Furthermore, an organization's process standards are tailored for each project to develop their own defined processes, as indicated in the upper right of Fig. 4. Once this has been established, the organization can introduce procedures for measuring the actual process execution. At this managed level, the organization learns to predict trends in processes and product quality. This action is depicted in the lower left of Fig. 4. Finally, at the optimizing level, the entire organization is focused on continuous process improvement (upper left of Fig. 4).

\section{CAME TOOLS}

CAME tools are being developed by many organizations around the world. As discussed above, CAME tools automate and control the application development processes, enabling the method engineer to develop fast, fluid, and flexible processes. These tools should increase planning, management, and development efficiency by providing tighter controls over each development project as it evolves. Furthermore, CAME tools ensure that methods are designed 
to be reusable and can be continually revised and improved through integration of best practices from previous projects.

A CAME tool is typically used for process management in four distinct modes-defining the process, planning the project, delivering the project, and improving the process.

- Defining the process - method components are created based on specific enterprise needs and characteristics. This ensures a successful foundation for a project. New method components can also be added to the library. The focus is on reusability and the intent that the processes will be used by project teams.

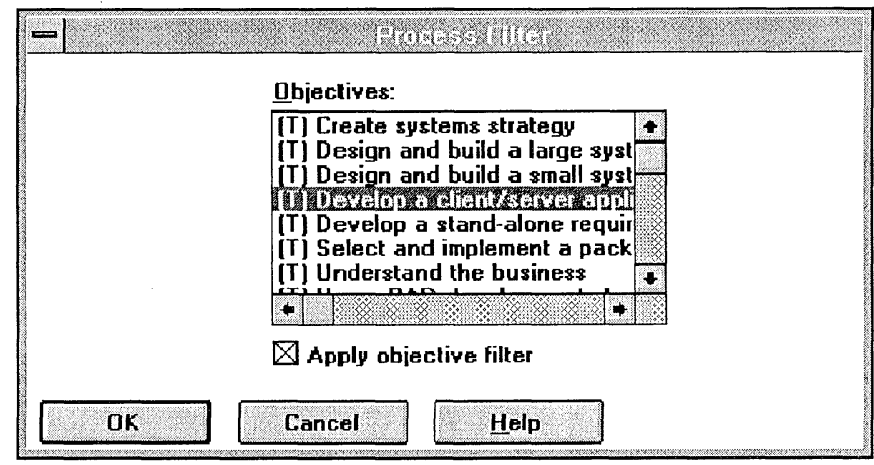

Figure 5 A screen that offers a choice of project objectives. Based on these objectives, the CAME tool can generate an appropriate methodology (Architect, James Martin \& Co.).

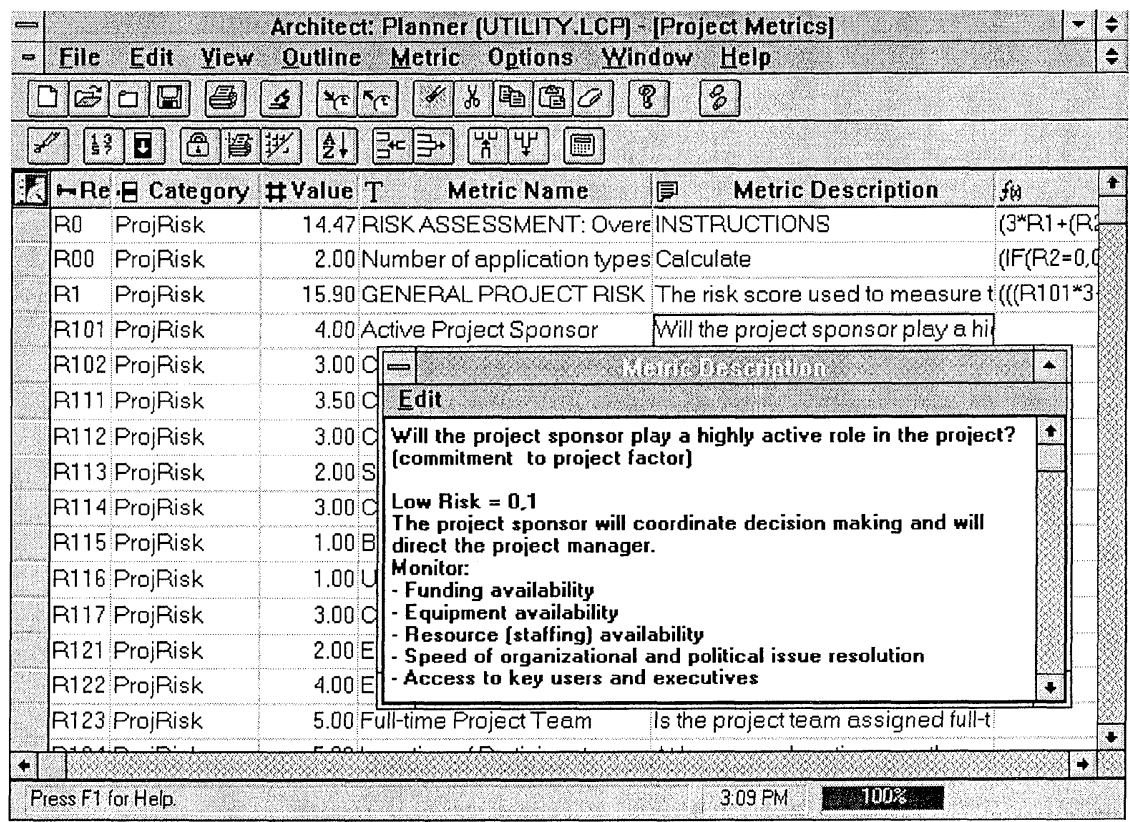

Figure 6 A screen that maintains and reports on various project metrics, such as duration and risk (Architect, James Martin \& Co.). 
- Planning the project-project managers are assisted in planning by assembling the necessary methodology for a particular project. Since the method base repository is constantly being improved from many different projects, project managers always have the most successful method components available to them. The methodology is tailored according to constraints of the individual project. Currently, both Ernst \& Young and James Martin \& Co offer such a tool. For example, Fig. 5 depicts a "process filter" screen from James Martin \& Company's CAME tool called Architect. This screen helps Architect to select the appropriate method segments based on the objectives selected in the right side of the window. Once the methodology is generated, the project can be estimated and its risk assessed. Figure 6 depicts an Architect project metrics screen.

- Delivering the project-system development work assignments can be assigned to individuals and to development tools. CAME tools can then guide the workflow of a project by ensuring that the right task is being completed by the right person, using the right tools.

- Improving the process-continuous improvement is key to process management. Using measurable quantitative feedback from each project, the method components used are reevaluated to determine what worked and what did not. Here, method components are modified, added, or deleted to reflect the best practices and lessons from SDLC projects.

\section{REFERENCES}

Coleman, Derek, Patrick Arnold, Stephanie Bodoff, Chris Dollin, Helena Gilchrist, Fiona Hayes, and Paul Jeremaes, Object-Oriented Development: The Fusion Method, Prentice Hall, Englewood Cliffs, NJ, 1994.

Harmsen, Frank, Sjaak Brinkkember, and Han Oei, "Situational Method Engineering for Information System Project Approaches," Methods and Associated Tools for the Information Systems Life Cycle, A. A. Verrijn-Stuart and T. William Olle, eds., Elsevier, Amsterdam, 1994, pp. 169-194.

Hutt, Andrew T. F., ed., Object-Oriented Analysis and Design: Comparison of Methods, Wiley-QED, New York, 1994a.

Hutt, Andrew T. F., ed.,Object-Oriented Analysis and Design: Description of Methods, WileyQED, New York, 1994b.

Jarke, Matthias, Klaus Pohl, Colette Roland, and Jean-Roch Schmitt, "Experience-Based Method Evaluation and Improvement: A Process Modeling Approach," Methods and Associated Tools for the Information Systems Life Cycle, A. A. Verrijn-Stuart and T. William Olle, eds., Elsevier, Amsterdam, 1994, pp. 1-27.

Martin, James, and James J. Odell, Object-Oriented Methods: Pragmatic Considerations, Prentice Hall, Englewood Cliffs, NJ, 1996.

Paulk, Mark C., Bill Curtis, Mary Beth Chrissis, and Charles V. Webber, "Capability Maturity Model, Version 2.1," IEEE Software, 10:4, 1993, pp. 18-27. 